\title{
Intracranial Cryptococcal Infection in HIV Positive Patient
}

\author{
Nabil Moatassim Billah, Touria Amil, Souad Chaouir \\ Military Hospital Instruction Mohammed V Rabat, Rabat, Morocco \\ Email: moatassimmbn@hotmail.com
}

Received 9 June 2013; revised 10 July 2013; accepted 18 July 2013

Copyright (C) 2014 by authors and Scientific Research Publishing Inc.

This work is licensed under the Creative Commons Attribution International License (CC BY). http://creativecommons.org/licenses/by/4.0/

(c) †) Open Access

\begin{abstract}
Cryptococcus neoformans is the most common fungal infection of the CNS. The majority of patients with cryptococcosis have preexisting immunodeficiency such as acquired immunodeficiency syndrome (AIDS). CT and/or MRI of the brain only occasionally detects abnormalities specifically related to this infection. We report a case of MR appearance of central nervous system cryptococcosis in a 33-year-old HIV positive man.
\end{abstract}

\section{Keywords}

Cryptococcus; HIV; CT; MRI

\section{Introduction}

Cryptococcus neoformans is an encapsulated yeast which is responsible for severe opportunistic meningoencephalitis mostly in patients with acquired immunodeficiency syndrome (AIDS).

It can infrequently be seen in immunocompetent patients as well. The organism enters via the respiratory tract and causes a spectrum of illness ranging from asymptomatic infection to severe illness, including pneumonia and disseminated infection involving multiple sites, including the central nervous system, eyes, and skin. The meningeal infection typically affects the basal brain and may also involve the adjacent parenchyma or may extend along the Virchow-Robin spaces. In some patients with cryptococcal meningitis, the neuroimaging studies show the presence of cryptococcomas and gelatinous pseudocysts. We report a case of MR appearance of central nervous system cryptococcosis in a 33-year-old HIV positive man.

\section{Case Report}

A 49-year-old HIV positive man presented in the emergency section of our hospital with altered sensorium of 4 days durations with complaints of headache and fever of 2 months duration with a few episodes of vomiting. 
Three weeks before admission, he developed a sudden onset of weakness on the left half of his body.

On examination, the patient was febrile. A neurological examination showed drowsiness and positive Kernig's sign. Cranial nerve functions and sensory functions were intact. A motor system examination showed leftsided hemiparesis.

An examination of cerebrospinal fluid showed white cells 4 cells $/ \mu \mathrm{L}$ lymphocytes, proteins $66 \mathrm{mg} / \mathrm{dl}$ and glucose $85 \mathrm{mg} / \mathrm{dl}$, India ink stain was positive for capsulated round yeast cells and mucicarmine positive capsule. A cryptococcal antigen test by latex agglutination kit (CALAS, Meridian Bioscience) was positive. A CSF culture on Sabouraud's dextrose agar yielded growth of C. neoformans that was nonfermentative, hydrolyzed starch, assimilated inositol and produced urease. Concanavine-glycine thymol blue agar was used to discriminate C. gattii from C. neoformans isolates.

The absolute CD4 count was 280 cells/ $\mu$ l. Distribution of the different serum immunoglobulins (IgG, IgM, IgA and IgE). The total leukocyte count was within normal limits, as well as the absolute neutrophil, eosinophil and basophil counts. The patient haved hypergammaglobulinemia as typically seen in patients infected with HIV.

The MR image showed small cystlike clusters in the right periventricular white matter. These were hypointense on T1-weighted, hyperintense on T2-weighted images and hypointense on T2 FLAIR. Some of the cysts demonstrated restricted diffusion, without postcontrast enhancement (Figure 1).

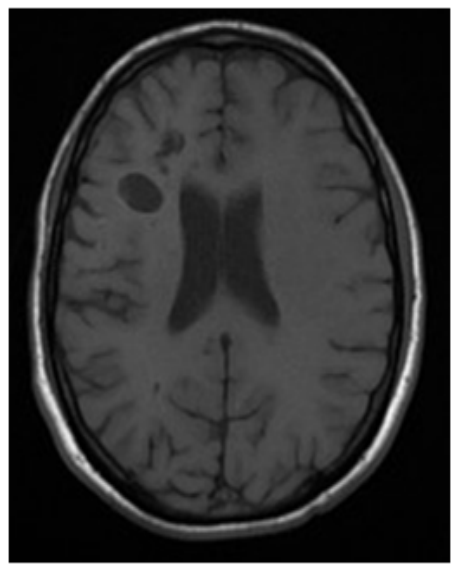

(a)

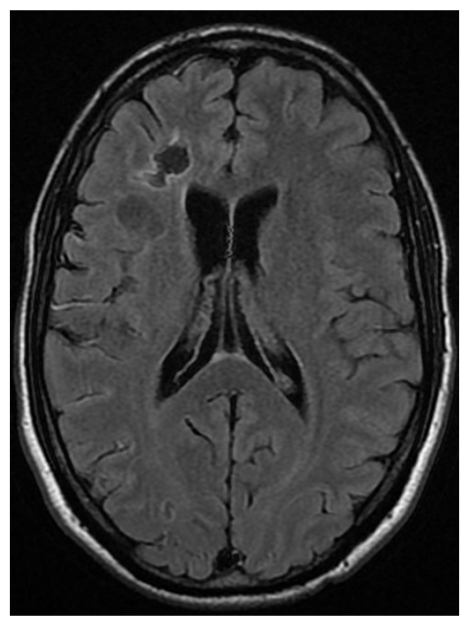

(c)

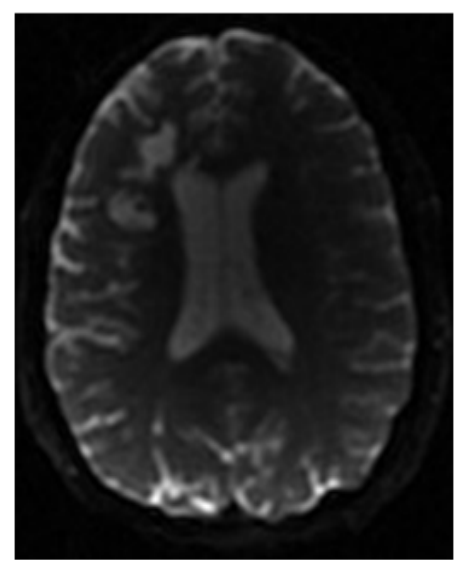

(b1)

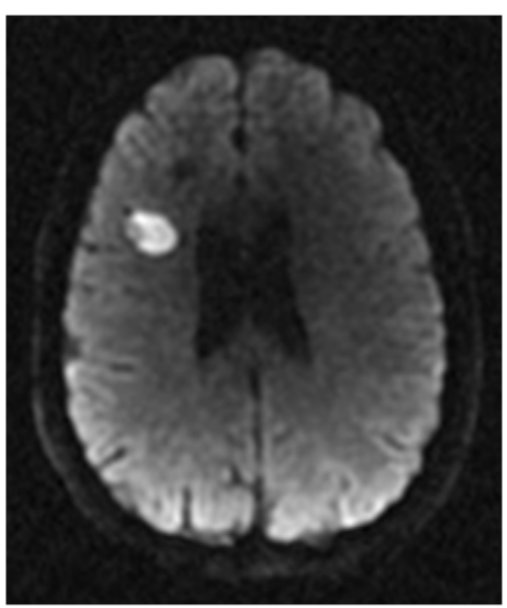

(d)

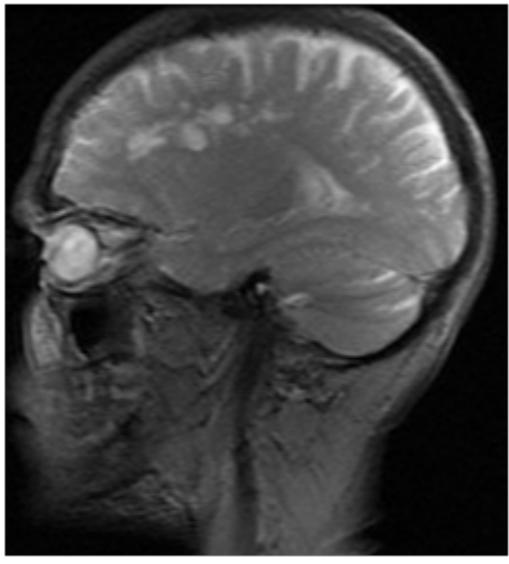

(b2)

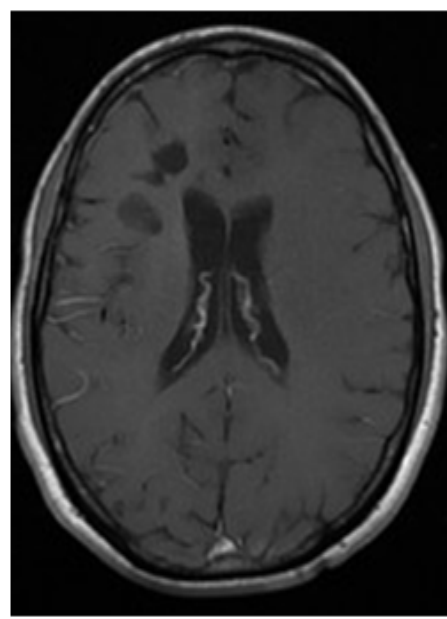

(e)

Figure 1. Brain MR imaging in a 33-year-old man. (a) Axial T1-weighted MR image shows a cysts hypointense lesions in the right periventricular white matter; Axial (b1) and Sagital (b2) T2-weighted MR image shows a cysts hyperintense lesions in the right periventricular white matter; (c) Axial fluid-attenuated inversion recovery image reveals dilated hyperintense Virchow-Robin spaces; (d) Axial diffusion-weighted imaging shows a restricted diffusion in one of the cysts lesions; (e) Enhanced axial T1-weighted image. Absence of meningeal enhancement. 


\section{Discussion}

The causative agent of cryptococcosis is the ubiquitous encapsulated fungus Cryptococcus neoformans [1]. Two pathogenic varieties are known. C. neoformans variety neoformans which, though worldwide in distribution, is found primarily in temperate areas, and C. neoformans variety gattii is found in the tropical and subtropical regions of Australia, Africa, America and Southeast Asia.

Old, dried pigeon droppings are the main reservoir of variety neoformans, while variety gattii has been isolated from Eucalyptus camaldulensis and more recently, from E. tereticornis [2], but has not been proven to exist in pigeon droppings. C. neoformans variety neoformans is most frequently found in immunosuppressed patients, whereas C. neoformans variety gattii manifests more typically with a granulomatous inflammatory response in immunocompetent patients, which results in chronic disease [3] [4].

Though HIV infection is presently the most common predisposing factor, other conditions that similarly alter host defenses, particularly cell-mediated immunity (CMI), can lead to severe cryptococcosis. Such conditions include collagen vascular disease, chronic corticosteroid therapy, sarcoidosis, Hodgkin's lymphoma, chronic lymphocytic leukemia, diabetes mellitus and immunosuppressive therapy aimed at $\mathrm{T}$ cells [5]. Epidemiological analysis revealed a male predominance. Among men and women, the average age showed differences in agreement with previous reports, suggesting that the average age is higher in women. Although rarely, cryptococcosis can affect children [6].

Central nervous system involvement is secondary to haematogenous spread, and usually results from a reactivation of a prior pulmonary infection. This predilection is because of the absence in the CSF of soluble anticryptococcal factors present in serum, the lack of significant activation of complement in the CSF, and the abundance of excellent substrate for more virulent yeasts. Extrapulmonary and extraneural manifestations were observed in $54 \%$ of patients. The following body sites are considered to be manifestations of disseminated disease: lymph node (lymphadenopathy), head and neck (involvement of oral cavity), skin and gastrointestines (hepatic) [6].

Headache is the most common symptom, but patients may also present with meningeal signs, confusion, blurred vision, and, rarely focal deficits [7] [8].

From the imaging standpoint, both CT and MR imaging are very informative, with the latter having very high sensitivity for the detection and characterisation of lesions. The spectrum of MR and CT abnormalities described in CNS cryptococcosis ranges from no abnormality to meningeal enhancement, ventriculomegaly, and meningoencephalitis in the form of either pseudocysts in the basal ganglia or granulomatous lesions [9] [10].

Meningitis is the most common manifestation, involving the leptomeninges, mostly the base of the brain with distension of the subarachnoid spaces by mucoid gelatinous exudates. Contrast enhancement is absent or minimal in patients with AIDS, due to impaired cell mediated immunity and inhibition of phagocytosis by the mucoid capsule [11].

The avascular pseudocysts are seen as well circumscribed, round to oval hypointensities on T1-weighted images and hyperintensities on T2-weighted images, which fail to enhance after administration of contrast medium. These are believed to represent dilated Virchow-Robin spaces filled with budding cryptococci enmeshed within a gelatinous material.

Granulomatous lesions enhance at contrast medium administration and are hyperintense on T2-weighted images. The development of these granulomatous lesions depends on the host immune response, and it is uncommon in AIDS patients. On imaging, CT of intracerebral cryptococcal granulomas has been reported as low-density lesions with or without homogeneous contrast enhancement or as isodense lesions with ring enhancement [12].

On diffusion-weighted images, there may be restricted diffusion in some of the lesions due to the high viscosity of their contents. The diffusion characteristics of cryptococcomas may resemble necrotic brain tumour, with the centre of the lesion showing no restricted diffusion [13].

MRI spectroscopy performed with a single voxel in the lesion demonstrated biochemical changes compatible with a focal inflammatory process with deficit of $\mathrm{N}$-acetyl-aspartate, and a small increased in the peak of choline. Chang et al. demostreted that The MR spectroscopy of cryptococcoma typically shows decreases in all the major cerebral metabolites like NAA, creatine, choline, myo-inositol, and the presence of a moderate amount of lipids in comparison to normal brain parenchyma. Lactate is rarely observed in cryptococcoma. Compared to toxoplasma and lymphoma, cryptococcoma shows a lesser amount of lipid and lactate [14]. 
Diagnosis can be made with Indian ink staining, culture or antigen tests on cerebrospinal fluid (CSF) obtained by lumbar puncture [15].

Patients with cryptococcal meningitis are usually treated with the antifungal drug amphotericin B.

\section{References}

[1] Levitz, S. (1991) The Ecology of Cryptococcus Neoformans and the Epidemiology of Cryptococcosis. The Journal of Infectious Diseases, 13, 1162-1169.

[2] Pfeiffer, T. and Ellis, D. (1992) Environmental Isolation of Cryptococcus Neoformans from Eucalyptus Tereticornis. Journal of Medical and Veterinary Mycology, 30, 407-408. http://dx.doi.org/10.1080/02681219280000541

[3] Drouet, A., Amah, Y., Pavic, M., Gérome, P., Meyer, X. and Debourdeau, P. (2005) Subacute Meningoradiculomyeloencephalitis due to Cryptococcosis Infection. La Revue de Médecine Interne, 26, 403-408. http://dx.doi.org/10.1016/j.revmed.2004.12.005

[4] Deus-Silva, L., Costa, A., Bevilacqua, J., et al. (2004) Meningoradiculitis Due to Cryptococcus Neoformans in an Immunocompetent Patient. Arq Neuropsiquiatr, 62, 147-149. http://dx.doi.org/10.1590/S0004-282X2004000100026

[5] Schmutzhard, F., Boongird, P., Gerstenbrand, F., et al. (1990) Is Cryptococcal Meningoencephalitis in Tropics a Distinct Entity? A Retrospective Study from Thailand. Tropical and Geographical Medicine, 42, 133-139.

[6] Gazzoni, A.F., Severo, C.B., Salles, E.F. and Severo, L.C. (2009) Histopathology, Serology, and Cultures in the Diagnosis of Cryptococcosis. Revista do Instituto de Medicina Tropical de São Paulo, 51, 255-259. http://dx.doi.org/10.1590/S0036-46652009000500004

[7] Harris, D.E. and Enterline, D.S. (1997) Fungal Infections of the Central Nervous System. Neuroimaging Clinics of North America, 7, 297-320.

[8] Galanis, E., Hoang, L., Kibsey, P., Morshed, M. and Phillips, P. (2009) Clinical Presentation, Diagnosis an Management of Cryptococcus gattii Cases: Lessons Learned from British Columbia Can. Canadian Journal of Infectious Diseases \& Medical Microbiology, 20, 23-28.

[9] Kovoor, J.M., Mahadevan, A., Narayan, J.P., Govindappa, S.S., Satishchandra, P., Taly, A.V. and Shankar, S.K. (2002) Cryptococcal Choroid Plexitis as a Mass Lesion: MR Imaging and Histopathologic Correlation. American Journal of Neuroradiology, 23, 273-276.

[10] Marcelo, C., María, F. and Ricardo, N. (2008) Magnetic Resonance Imaging Findings in AIDS with Central Nervous System Cryptococcosis. Revista Iberoamericana de Micología, 25, 211-214.

[11] Ruiz, A., Post, M.J. and Bundschu, C.C. (1997) Dentate Nuclei Involvement in AIDS Patients with CNS Crypococcosis: Imaging Findings with Pathologic Correlation. Journal of Computer Assisted Tomography, 21, 175-182. http://dx.doi.org/10.1097/00004728-199703000-00003

[12] Kamezawa, T., Shimozuru, T., Niiro, M., Nagata, S. and Kuratsu, J. (2000) MRI of a Cerebral Cryptococcal Granuloma. Neuroradiology, 42, 441-443. http://dx.doi.org/10.1007/s002340000321

[13] Patro, S.N., Kesavadas, C., Thomas, B., Kapilamoorthy, T.R. and Gupta, A.K. (2009) Uncommon Presentation of Intracranial Cryptococcal Infection Mimicking Tuberculous Infection in Two Immunocompetent Patients. Singapore Medical Journal, 50, e133

[14] Chang, L. and Ernst, T. (2005) Imaging of Intracranial Infections in the Immunocompromised host. In: Latchaw, R.E., Kucharczyk, J. and Moseley, M.E., Eds., Imaging of the Nervous System: Diagnostic and Therapeutic Applications. Elsevier Mosby, Philadelphia, 945-947.

[15] Teo, Y.K. (2010) Cryptococcal Meningoencephalitis with Fulminant Intracranial Hypertension: An Unexpected Cause of Brain Death. Singapore Medical Journal, 51, e133. 